\title{
Management of Patients with Node Positive Prostate Cancer at Radical Prostatectomy and Pelvic Lymph Node Dissection: A Systematic Review
}

\author{
Giancarlo Marra1, Massimo Valerio², Isabel Heidegger ${ }^{3}$, Igor Tsaur ${ }^{4}$, Romain \\ Mathieu ${ }^{5}$, Francesco $\mathrm{Ceci}^{6}$, Guillaume Ploussard ${ }^{7}$, Roderick C.N. van den Bergh", \\ Alexander Kretschmer ${ }^{9}$, Constance Thibault ${ }^{10}$, Piet Ost ${ }^{11}$, Derya Tilki ${ }^{12,13}$, Veeru \\ Kasivisvanathan ${ }^{14,15}$, Marco Moschini ${ }^{16}$, Rafael Sanchez-Salas ${ }^{16}$, Paolo Gontero ${ }^{1}$, \\ R. Jeffrey Karnes ${ }^{17}$, Francesco Montorsi ${ }^{18}$ and Giorgio Gandaglia ${ }^{18}$ on behalf of the \\ EAU-YAU Prostate Cancer Working Party
}

\begin{abstract}
1'Department of Urology, San Giovanni Battista Hospital, University of Turin, Turin, Italy 2Department of Urology, CHUV Lausanne, Lausanne, Switzerland

${ }^{3}$ Department of Urology, Medical University Innsbruck, Innsbruck, Austria

${ }^{4}$ Department of Urology and Pediatric Urology, Mainz University Medicine, Mainz, Germany

${ }^{5}$ Department of Urology, CHU Rennes, Rennes, France

${ }^{6}$ Nuclear Medicine, Department of Medical Sciences, University of Turin, Italy

${ }^{7}$ Department of Urology, La Croix du Sud Hospital, Toulouse, France and Institut Universitaire du Cancer Toulouse - Oncopole, Toulouse, France

${ }^{8}$ Department of Urology, Antonius Hospital, Utrecht, The Netherlands

${ }^{9}$ Department of Urology, Ludwig-Maximilians-University of Munich, Munich, Germany ${ }^{10}$ Department of Oncology, Hopital Europeen Georges Pompidou, Paris, France

${ }^{11}$ Department of Radiation =ncology, Ghent University Hospital, Ghent, Belgium ${ }^{12}$ Martini-Klinik Prostate Cancer Center, University Hospital Hamburg-Eppendorf, Hamburg, Germany ${ }^{13}$ Department of Urology, University Hospital Hamburg-Eppendorf, Hamburg, Germany

${ }^{14}$ Division of Surgery and Interventional Science, University College London, London, UK

${ }^{15}$ Department of Urology, University College London Hospital, London, UK

${ }^{16}$ Department of Urology, Institut Mutualiste Montsouris, Paris, France

${ }^{17}$ Department of Urology, Mayo Clinic, Rochester, MN, US

${ }^{18}$ Division of Oncology/Unit of Urology, Urological Research Institute, IRCCS Ospedale San Raffaele, Milan, Italy
\end{abstract}

\section{Corresponding Author:}

Giancarlo Marra, MD

Email: drgiancarlomarra@gmail.com

Department of Urology

San Giovanni Battista Hospital and University of Turin

C.so Bramante 88/100

10100 Turin, Italy 


\section{Abstract:}

Introduction: The optimal management of prostate cancer (PCa) patients with lymph node invasion ( $\mathrm{LNI}$ ) at radical prostatectomy (RP) and pelvic lymph node dissection (PLND) still remains unclear.

Objective: To assess the effectiveness of postoperative treatment strategies for pathologically node-positive PCa patients. The secondary aim was to identify the most relevant prognostic factors to guide the management of $\mathrm{pN} 1$ patients.

Evidence Acquisition: A systematic review was performed in January 2020 using Medline, Embase and other databases. A total of 5,063 articles were screened and 26 studies including 12,537 men were selected for data synthesis and included in the current review according to the PRISMA recommendations.

Evidence Synthesis: Ten-year biochemical recurrence (BCR)-, clinical recurrence (CR)-, cancer-specific (CSS)- and overall (OS)- survival rates ranged from $28 \%$ to $56 \%, 70 \%$ to $92 \%, 72 \%$ to $98 \%$ and $60 \%$ to $87.6 \%$, respectively. A total of 7,5 , and 6 studies assessed the oncologic outcomes of observation, adjuvant radiotherapy (aRT) or adjuvant androgen-deprivation (ADT), respectively. Initial observation followed by salvage therapies at the time of recurrence represents a safe option in selected patients with low disease burden. The use of aRT with or without ADT might improve survival in men with locally advanced disease and a higher number of positive nodes. Risk stratification according to pathological Gleason score, the number of positive nodes, pathological stage and surgical margins status is key for risk stratification and selection of the optimal postoperative therapy. Limitations of this systematic review are the retrospective design of the studies included and the lack of data on adverse events.

Conclusions: While the majority of men with $\mathrm{pN} 1$ disease would experience BCR after surgery, long-term disease-free survival has been reported in selected patients. Management options to improve oncologic outcomes include observation vs. adjuvant therapies such as aRT and/or ADT. Disease characteristics should be used to select the optimal postoperative management for pN1 PCa patients.

Patient Summary: Finding nodes positive prostate cancer after a radical prostatectomy often leads to high post-operative prostate specific antigen levels and is overall a poor prognostic factor. However, this does not necessarily translate into poor survival for all men. Management can be tailored to the severity of disease and options include observation, androgen deprivation therapy or radiotherapy. 


\section{Introduction}

Up to $15 \%$ of contemporary patients affected by clinically localized prostate cancer (PCa) harbor lymph node invasion ( $\mathrm{LNI}$ ) at final pathology after radical prostatectomy (RP) and pelvic lymph node dissection (PLND) [1]. The presence of LNI represents one of the most important prognostic factors for recurrence and cancer-specific mortality, where pN1 patients have a worse prognosis as compared to their counterparts with node-negative disease [2]. Nonetheless, the optimal management for node positive patients still remains unclear. The presence of LNI at final pathology represented an exclusion criteria in recent randomized trials (RCTs) aimed at assessing the role of postoperative radiotherapy (RT) in patients with adverse disease characteristics at RP [3]. Moreover, although a RCT specifically designed in the pN1 setting demonstrated that immediate androgen deprivation therapy (aADT) might be associated with a survival benefit, its results might not be generalizable to contemporary pN1 patients due to the inclusion of patients diagnosed in the pre-PSA era with high nodal burden $[4,5]$. Finally, despite the fact that all patients with LNI are categorized as $\mathrm{pN} 1$ regardless of the number and location of positive nodes according to the TNM classification, the long-term prognosis of this group is highly heterogeneous and varies significantly according to disease characteristics [6-13]. This, in turn, might have important implications for the selection of the most optimal postoperative management strategy.

Given the lack of level-1 evidence applicable to contemporary patients, we performed a systematic review of the available literature to summarize the evidence on the management of pN1 PCa patients at RP and PLND and to determine their optimal postoperative management. Moreover, we sought to assess prognostic factors that should guide multimodal management of $\mathrm{PCa}$ patients with $\mathrm{pN} 1$ disease after surgery.

\section{Methods}

An 'a priori' protocol was submitted to PROSPERO for registration on the $16^{\text {th }}$ of November 2019 (Receipt Number: 158801). The protocol is provided as a supplementary file. Study objectives were framed according to the PICO criteria. Our primary aim was to investigate the optimal management of non-metastatic $\mathrm{PCa}$ patients with $\mathrm{pN} 1$ disease at RP and PLND by assessing oncological outcomes according to different treatment modalities. Secondary aims were to identify relevant 
prognostic factors and risk categories/models to guide the management of pN1 patients.

A systematic web search was performed according to the Preferred Reporting Items for Systematic Reviews and Meta-analyses (PRISMA) guidelines on January $28^{\text {th }}$ 2020 through the Ovid platform with no time restrictions using AMED (Allied and Complementary Medicine), HMIC (Health Management Information Consortium), Embase and Medline. The terms "pN1", "pN+" and ("positive" AND "lymph node") were pooled together with the boolean operator OR. Results were then pooled together with the terms "prostate cancer" and "radical prostatectomy" using the boolean operator AND. Web Search was implemented by manual search (authors consultation and references of web-search included articles). Two authors (G.M. and G.G.) independently screened all items. Disagreements were resolved through consensus or by consultation with a third author (M.V.).

Full-text publications in English were considered. The study population comprised male patients with histologically proven $\mathrm{PCa}$ and LNI at final pathology. The treatment options of interest included observation with or without salvage therapies, adjuvant RT (aRT) and/or ADT or other systemic therapies after surgery. The primary outcome was cancer-specific (CSS) survival. Secondary outcomes were represented by biochemical recurrence (BCR)-, clinical recurrence (CR)-, and overall survival (OS). We included RCTs and prospective series with more than 50 cases without restrictions. Retrospective series were included if: i) included $\geq 100$ patients with pN1 disease pathologically proven following RP and PLND; ii) provided at least details on PLND type being performed or number of nodes removed; iii) included non-metastatic patients; iv) reported oncological outcomes of pN1 patients according to different treatment strategies or the role of prognostic factors assessed at multivariate analysis adjusted for treatment strategy. Retrospective studies were excluded if: i) included only $\mathrm{cN}+$ patients; ii) did not provide baseline features of the pN1 group; iii) did not specify the exclusion of metastatic patients from the study cohort; iv) assessed the role of salvage RP or salvage lymphadenectomy; v) reported early outcomes of series being updated at a later time without providing additional information (studies using the same cohort of previously included works but providing additional analysis/information were included).

Risk of bias and study quality was assessed according to EAU recommendations for performing systematic reviews and meta-analysis [14]. The Cochrane risk of bias 
assessment tool V2.0 was used for RCTs and the Quality Appraisal tool for case series using a Modified Delphi technique for retrospective studies [15], as previously performed [16]. Complications were classified according to Clavien-Dindo and adhering to EAU Guidelines on complications [17]. Data extraction form is provided in Supplementary Material 1.

\section{Results}

\subsection{Features of studies included in the systematic review}

The PRISMA flowchart is depicted in Figure 1. We included 26 studies $(n=23$ retrospective series and $n=3$ RCTs) reporting results of 12,537 patients undergoing RP and PLND between 1982 and 2017 (Table 1). Five institutions included the same cohorts in multiple studies, with three institutions providing patients in 11, 6 and 4 studies respectively (Supplementary Material 2). When excluding multiple entry data, the actual number of patients assessed was 4,067. Only two studies used a nomogram to select patients for PLND at the time of surgery $[18,19]$. Nine series did not specify the imaging modality used for patient selection and/or follow up, 12 used different combinations of bone scan, abdominopelvic CT and/or MRI and/or US, and chest-Xray. Five series used PET-choline (only one study detailed the exact number of men undergoing PET/CT-choline [20]). Among the fourteen studies including adjuvant RT as a treatment option, seven performed concomitant lifelong adjuvant ADT whilst seven did not specify adjuvant ADT duration. Cancer-specific and overall survival/mortality were primary outcomes in 16 and 13 studies, respectively. Metastasis free survival (MFS), included as primary outcome in 5 studies, was assessed through imaging confirmation in 12 studies whilst 14 did not specify the criteria used. Biochemical recurrence (BCR) was primary endpoint in 13 studies and was defined using different cut-off values $(>0.2 \mathrm{ng} / \mathrm{mL}$ in $n=12,>0.4 \mathrm{ng} / \mathrm{mL}$ in $n=4,>0.1$ in $n=1$, depending on the year of surgery $n=1$ or not specifying any $n=8$, respectively). Quality of the studies and risk of bias assessment is displayed in Table 2. None of the included studies detailed complications/side effects of adjuvant/salvage treatments.

\subsection{Patients' Features}

Overall baseline and pathological features of 22 cohorts ( $n=26$ studies) are shown in Table 3. Fifteen studies described the type of PLND, with extended or standard PLND being used in thirteen. Only two cohorts [21,22] did not report the number of nodes 
removed, which was always $\geq 10$ in the remaining studies with the exception of Cai et al. (mean of 5.2 nodes removed) [23]. The median number of positive nodes was reported in fifteen cohorts and was 3 and 2 in one and seven series respectively, and lower in the remaining cohorts. All series had a median age $<68$ years old with the exception of one reporting a mean age of 72.6 years [23]. Pre-operative PSA was $>15 \mathrm{ng} / \mathrm{mL}$ in three series $[4,5,24,25]$. Of twenty cohorts detailing pathological Gleason score 4,339 on 10,205 men (42.5\%) had Gleason score $\geq 8$. Of eighteen series detailing pathologic stage, 6,752 on 9,698 patients $(69.6 \%)$ had $\geq p$ T3b stage. In the nineteen series detailing margin status 5,363 on 10,108 men $(53.0 \%)$ had positive margins. Median follow up ranged from 16.1 months [26] to 17.6 years [10] with 5 series having a follow up $<50$ months.

\subsection{Randomized controlled trials}

Messing and colleagues randomized 98 patients with LNI at RP either to immediate ADT or observation. Men undergoing observation received salvage treatment when experiencing local and/or distant progression. The study did not achieve the planned sample size of $n=220$ patients and was closed due to accrual issues related to PSA introduction in clinical practice. At a median follow-up of 11.9 years, progression-free survival (53.2\% vs. $13.7 \%, \mathrm{p}<0.001)$, CSS (85\% vs. $51 \%, \mathrm{p}<0.001)$ and overall survival (64\% vs. $45 \%, p=0.04)$ were higher in the ADT arm compared to observation $[4,5]$. Another RCT investigated the use of mitoxantrone in addition to ADT for highrisk $\mathrm{PCa}$ and detailed results for the subgroup of patients having pN1 disease. No survival advantages were noted for pN1 patients included in the mitoxantrone arm compared to those managed with ADT alone (10-year OS: $81 \%$ in both arms). Moreover, the trial was prematurely closed due to safety issues as mitoxantrone increased the risk of leukemia and other malignancies [21]. Adverse events and baseline features of pN1 patients were not separately reported.

\subsection{Retrospective Series}

\subsubsection{Prognostic Factors}

Seventeen studies performed multivariate analysis to assess prognostic factors in $\mathrm{pN} 1$ patients (Table 4). Eleven [8,10,31,23-30], two [11,32] six [8,10,20,24,25,28], thirteen [7,10,33-35,11,18,20,24,25,27,29,32] and six [7,18,27,29,33,34] investigations evaluated prognostic factors for BCR, clinical recurrence, MFS, CSS and overall 
survival respectively. Apart for one study [26], age was not a significant predictor of any oncological outcomes whilst three on five studies evaluating its impact on overall survival found an association with elderly age $[10,27,33]$. PSA values were marginally associated with oncological outcomes [8,24-26] with the exception of one investigation reporting worse CSS for individuals with PSA levels higher than $40 \mathrm{ng} / \mathrm{mL}$ [18]. Only about half of the series assessing pathologic stage reported a correlation between this covariate and oncologic outcomes. Conversely, all the three studies $[10,18,33]$ investigating the association between stage and overall survival failed to show a significant impact. Five out of eight studies confirmed an association between positive margins and BCR. However, surgical margins were not associated with stronger oncologic outcomes such as metastases-free survival. Four out of nine and two out of four series did not show significant correlations between margin status and CSS or overall survival. Conversely, almost all available series recorded an association between pathological Gleason score and the risk of BCR (9/10), clinical $(1 / 1)$ or systemic (5/6) recurrence, CSS (12/12) and overall mortality (4/6). The number of positive nodes was associated with BCR, clinical and systemic recurrence in four out of eight, two out of two and three out of five series, respectively. Almost all (6/7) series found association between the number of positive nodes and cancer-related deaths. Only one out of three studies reported an association between this variable and overall survival [33]. One study proposed that a higher number of removed nodes was associated with improved CSS [35]. However, none of the remaining five series assessing the prognostic value of this variable reported an association with oncological or survival outcomes [10,20,25,27,33]. Amongst other factors, year of surgery [25,27,28,33,35], extranodal extension [26] and the side of LNI were not reported as significant predictors of oncologic outcomes. Conversely, positive associations were described for the size of nodal metastases [26,29,34] with BCR and survival; for nodal lymphovascular invasion [31] with BCR; for PSA persistence [32] with clinical recurrence and CSM.

Two series investigated prognostic factors in node positive patients experiencing CR and reported that men experiencing skeletal and visceral recurrences had worse CSS compared to their counterparts with a first metastasis in other sites (i.e., the lymph nodes) $[11,20]$. This applied also when considering multiple vs. single and early vs. late recurrences [11]. Shorter time between surgery and BCR and receiving a PET/CT- 
choline also represented independent predictors of clinical and systemic recurrence [20].

\subsubsection{Risk stratification according to multivariable models}

Four studies developed five different models, one for overall survival and four for CSS $[7,9,10,33]$ whilst another cohort was used for external validation of a CSS prediction nomogram [19] (Supplementary material 3). Two institutions included patients in four and three of these studies, respectively. Two additional studies developed models to predict BCR $[8,26]$. Abdollah and colleagues built different models based on a single cohort of 1,107 cases to predict CSS at 8 and 10 years in pN1 patients [7,33]. In their first study, a regression tree analysis was used to identify five risk groups (based on positive nodes, Gleason score, pathologic stage and margins) to predict the risk of dying from PCa itself. In the second work, two nomograms were developed, one for the overall population and another for those experiencing BCR with discrimination accuracies of $79.5 \%$ and $83.3 \%$ respectively [33]. The nomogram based on the overall population has been externally and independently validated in a multi-institutional cohort showing predictive accuracy inferior to what obtained at internal validation (65.8\% vs. $79.5 \%$, respectively). The reason for such a discrepancy might reside in differences amongst the development and validation cohorts related to the length of follow-up, the use of adjuvant therapies and disease characteristics. Despite the suboptimal calibration, the use of the nomogram was associated with a net benefit at decision curve analysis [19].

Longer term CSS was assessed by a single center series, which defined a risk-score to determine low-, intermediate- and high-risk CSM categories using Gleason score, margin status and the use of aRT. This classification stratified patients in three groups with 20 -year CSM rates of $19.1 \%$ vs. $34 \%$ vs. $46 \%$ for low-, intermediate- and highrisk, respectively [10]. Other authors developed different models to predict short term BCR (median follow up of 16.1 months) based on 484 patients with the final model also including extranodal extension and largest node metastases size [26]. Finally, Touijer et al.[8] estimated the conditional BCR-free probability depending on the number of years free from BCR from RP in men undergoing observation and reported that the 10 -years BCR free probability ranged from $49 \%$ to $81 \%$ for those not recurring within the first and fifth year after RP, respectively. 


\subsubsection{Postoperative management options}

\section{Observation}

Table 4 depicts the oncological outcomes of node positive patients according to the management strategy. Six series ( $n=7$ studies) reported the outcomes of 1,377 patients managed with observation following RP and PLND. In three series observation mainly consisted of periodical follow-up until BCR (defined as PSA values $>0.2 \mathrm{ng} / \mathrm{mL}$ ), when patients were offered salvage treatments such as ADT and/or RT $[8,24,28]$ whilst in the other three cohorts the authors did not specify criteria for salvage treatments [36] or offered salvage options in case of clinical [30] or symptomatic [29] progression, respectively. Overall, BCR was experienced by $62 \%$ of the patients with reported BCR-free survival rates ranging from $43 \%$ at 4 years [28] to $28 \%$ at 10 years [8]. Cancer-related and overall deaths were reported in $12.2 \%$ and $21.2 \%$ of patients respectively with CSS rates ranging from $78 \%$ at 5 years to $72 \%$ at 10 years and OS from $75 \%$ at 5 years to $60 \%$ at 10 years. Baseline features of patients in the observation group are depicted in Supplementary Material 4. The majority of patients managed with initial observation after surgery had favorable disease characteristics, where $63 \%$ of them had only one positive node. Moreover, pathologic Gleason score $<8$ and negative surgical margins were reported in $64 \%$ and $62.5 \%$ of the cases, respectively.

\section{Adjuvant Radiotherapy with/without adjuvant ADT}

Four series ( $n=5$ studies) reported results for aRT $(n=1,271$ men) with $(n=958)$ or without $(n=100)$ concomitant ADT, whereas for 213 patients the authors did not specify if concomitant ADT was used [7,19,22,28,33]. All individuals included in these cohorts received whole pelvis aRT which included the prostatic bed and pelvic nodes. BCR was not reported whereas one series detailed CR-free survival [22]. Cancer-specific survival ( $n=3$ series) ranged from $72.4 \%$ at 8 years to $86.7 \%-98 \%$ at 10 years. Overall survival ranged from $81 \%$ to $87.6 \%$ at 10 years $[7,19,22,33]$. Patients undergoing aRT generally showed more aggressive features compared to the observation group; 3 or more positive nodes were present in $30.1 \%$ and the majority had Gleason Score $\geq 8$ (53.3\%), pT3b (66.3\%) and positive margins (66.8\%) (Supplementary Material 4).

\section{Adjuvant Androgen Deprivation Therapy}


Adjuvant ADT results were reported by five retrospective series ( $n=6$ studies) including 1,319 men $[7,19,23,28,31,33]$. Biochemical recurrence-free survival ranged from $61 \%$ at 5 years to $56 \%$ at 10 years $[7,19,31,33]$. No studies detailed CR or metastases-free survival and only two series 8 and 10 years CSS being $69 \%$ and $82.3 \%$ respectively $[7,19,33]$. The sole group reporting overall survival described an 8 years rate of $75.1 \%$ [7,33]. Patients undergoing aADT alone typically had aggressive disease characteristics with 3 or more positive nodes in $39 \%$ of the cases.

\section{Comparative studies}

Three series compared aRT+/-ADT vs. ADT alone. One study compared initial observation ( $n=505, n=258$ receiving $s R T$ after $B C R$ ) vs. aRT $\pm A D T$ and reported inferior BCR- and metastases-free survival rates in men managed with initial observation compared to aRT. These results were confirmed after propensity score matching [28]. When considering CSS ( $n=2$ series), one study reported that aRT was not characterized by better CSS rates at univariable analyses. However, when performing multivariate analysis, ADT alone showed a significant increased risk of CSM compared to aRT with ADT (OR 2.72, 95\% Cl 1.62-4.55, p<0.001) [7,33]. Other series failed to show advantages associated with the use of aRT, where similar CSS rates were reported (8 year CSS ADT 69\% vs. $72.4 \%$ for ADT vs. aRT, respectively [19]; 10 year CSS $82.3 \%$ vs. $86.7 \%$ for ADT vs. aRT, respectively [7,33]) [19].

Several authors proposed that the benefits of aRT with or without concomitant ADT might differ according to disease characteristics. Abdollah et al. [7] developed risk categories for CSM and reported that aRT with ADT was associated with improved CSS and OS rates only in men with intermediate-risk (namely, patients with two or fewer positive nodes, Gleason score 7 to 10, and pT3b/pT4 or positive margins; HR $0.30-95 \% \mathrm{Cl}$ 0.14-0.64; $\mathrm{p}=0.002$ and $\mathrm{HR}$ 0.42, 95\% Cl 0.25-0.70, $\mathrm{p}=0.001$, respectively), and high-risk (namely, patients with 3 to 4 positive nodes; HR $0.2195 \%$ Cl 0.06-0.79; $p=0.02$ and $\mathrm{HR} 0.32,95 \% \mathrm{Cl} 0.12-0.83, p=0.02$, respectively) disease and not in the remaining ones. These results were externally validated in a cohort of $\mathrm{n}=3,158 \mathrm{pN} 1$ patients included in the SEER registry [7]. Similarly, Touijer et al. reported that in their series of 1,338 node positive patients aRT with ADT was associated with improved overall survival as compared to ADT alone or observation. However, the magnitude of the effect of aRT with ADT compared to observation or ADT alone on survival varied according to individual disease features. In this context, 
the authors developed a 0 to 8 score and reported that higher risk patients were the ones who were more likely to benefit from aRT as compared to men at lower risk of mortality according to disease characteristics (i.e., 0 points $=10$ year overall mortality $17.0 \%$ without aRT vs. $12.3 \%$ with ADT+aRT: $4.7 \%$ gain; 8 points=10 year overall mortality $71.1 \%$ without vs. $31.3 \%$ with aADT+aRT: $+39.8 \%$ gain) [9].

Finally, one study recently assessed the need for adding ADT to aRT in a series of 372 node positive patients. When comparing aRT with ADT vs. aRT alone, no significant differences were found in CSS, overall survival and clinical progression, even when performing matched pair and sensitivity analysis. These results were confirmed at multivariable analyses and question the effectiveness of concomitant ADT at the time of aRT [22].

\section{Discussion}

Although a substantial proportion of $\mathrm{PCa}$ patients treated with RP and PLND for clinically localized PCa eventually harbor a node positive disease at final pathology, the optimal postoperative management of these men is still unknown. Hence, we performed a systematic review to investigate the optimal management of pN1 patients at RP and PLND according to disease features.

Our findings are several-fold. First, a non-negligible proportion of men would experience long-term disease-free survival even when managed without additional cancer therapies. For example, retrospective series reported that up to one out of three patients managed with initial observation would not experience BCR at 10-year followup. Moreover, the 10-year CSS and OS rates exceeded $70 \%$ for selected nodepositive patients with favorable features. Taken together, these findings highlight that selected men with node positive disease should not be considered as affected by a systemic disease and a close follow-up based on PSA testing can represent a safe option. Nonetheless, it should be noted that the majority of pN1 patients typically harbor other aggressive characteristics at final pathology such as seminal vesicles invasion, Gleason score $\geq 8$ and positive surgical margins. All these factors yield an increased risk of worse oncological outcomes per se [37-39]. Within this high-risk context, survival rates do not markedly differ compared to previous reports focused on node negative patients with locally aggressive disease [40] and are higher compared to what reported for men with oligometastatic PCa treated with RP [41]. 
Second, pN1 PCa represents a multifaceted group rather than a single entity [7]. Amongst the numerous clinical and/or pathological variables of pN1 patients investigated in the included studies, Gleason score and number of positive nodes seem the most relevant for the identification of men more likely to experience CSS and overall survival. Similarly, pathological stage and surgical margins may have a prognostic value in a pN1 context. Four different studies developed cancer-specific or overall survival prognostic models. Overall, the results of these series clearly confirm the heterogeneity of pN1 PCa, where not all node positive patients may be affected by systemic disease. This is particularly true when considering contemporary men with a low nodal burden. On the other hand, in these individuals the risk of disease progression might be driven by the presence of aggressive disease features at the level of the prostate. As such, adjuvant therapies might play a role to improve oncologic control. Different strategies have been proposed for the postoperative management of pN1 PCa patients including observation, aADT and aRT. Although all these postoperative seem to yield acceptable outcomes, patient selection is key to optimize oncologic results. For example, the majority of series assessing oncologic outcomes of initial observation in pN1 patients include well-selected patients with a Gleason score $<8$ and a low number of positive nodes. Conversely, available evidence does not support the use of initial observation in men with a high nodal burden or more aggressive disease features at final pathology [8]. The potential role of immediate ADT after surgery has been tested in a randomized controlled trial specifically focused on pN1 patients [4]. However, the results of this study are hardly applicable to contemporary patients due to differences in the nodal burden, use of PSA as well as timing of salvage. In line with these thoughts, more recent retrospective series investigating observation versus ADT did not report any marked differences in oncologic outcomes $[9,28]$. Finally, aRT has been proposed to improve survival in pN1 patients with a relatively low nodal burden and aggressive pathologic disease features at the level of the prostate. When delivered to properly selected patients, this treatment option achieved long-term disease-free survival in up to half of them. Moreover, less than one on four men experiences cancer or other cause-related-death at 10-year follow-up.

From a clinical perspective, current evidence supports treatment choice tailored according to a risk and patient-adapted strategy to maximize the balance between oncological control and side effects. Observation followed by salvage treatments might 
represent a reasonable upfront option in many patients with a lower risk of recurrence whilst aRT (prostatic fossa plus pelvis) and/or ADT could be considered in those with aggressive disease features (Supplementary Figure 1). Recent evidence from RCTs failed to show a survival benefit of adjuvant versus early salvage $R T$ for high-risk nodenegative PCa patients at the cost of worse functional outcomes and side effects in the aRT group [3][42]. However, the presence of a node positive disease represented an exclusion criterion for these trials and further randomized trials are needed to determine whether these results might be applicable to the pN1 scenario. In the context of aRT, concomitant aADT duration also lacks of evidence, with the majority of the studies performing lifelong aADT or not stating its duration, and certainly needs to be further explored.

Despite our work representing the first systematic review assessing the best management of pN1 patients, some limitations should be highlighted. First, the level of evidence is mainly based on retrospective series and with recruitment in the two available RCTs being stopped early before reaching the planned sample size [4,21]. Second, none of the studies reported adjuvant treatment-related complications and side effects. Hence, the risk-benefit ratio of adjuvant treatments remains speculative and cannot be appropriately estimated. Third, many studies derive from few institutions which used their patients' cohorts multiple times. When adjusting for possible patients re-inclusion, all current evidence on pN1 disease is based on approximately 4,000 men. Fourth, though our search did include for series published from contemporary practice, it also included series of patients published more than two decades ago. Staging and selection criteria included PET-Choline sporadically and none of the series performed pre-operative PET-PSMA. It is noteworthy that none of the studies evaluated included upfront staging using PSMA-PET. In a first line setting, increasing PSMA availability may favor a shift towards lower frequency of positive nodes and, in case of $\mathrm{pN} 1$, in lower disease burden; in BCR setting it may offer improved and quicker information on disease location and may guide the type and site of adjuvant and/or salvage treatments. Given recent evidence in favor of a new staging pathway for high-risk $\mathrm{PCa}$, the paradigm of $\mathrm{pN} 1$ disease after RP and LAD will likely be revolutionized in the coming years [43]. Molecular biomarkers and genomics may also potentially increase risk stratification ability favoring patienttailored approaches for pN1 disease [44,45]. Although none of the available studies assessing the role of biomarkers in selecting candidates for additional cancer 
therapies were specifically developed in cohorts of node-positive patients, Dalela et al recently showed that the inclusion of the Decipher score in a risk tool aimed at selecting candidates for adjuvant radiotherapy might improve its accuracy [44]. Therefore, further studies are needed in this setting to better elucidate the role of biomarkers in patients with pN1 disease

Trials comprising novel staging modalities, biomarkers and assessing new antiandrogen drugs, including apalutamide and abiraterone acetate, are ongoing (NCT04134260). These studies will likely further shed light on the management of patients being found with pN1 after radical prostatectomy.

\section{Conclusions}

Pathologically positive nodes patients are characterized by aggressive PCa features. Nonetheless, oncological control and survival rates are promising, with a significant proportion of patients remaining disease-free. Different management strategies are available including initial observation, aRT and/or aADT. The choice of these treatments may be tailored according to prognostic factors and/or risk stratification tools. Initial observation may be considered for lower risk features, including undetectable post-operative PSA, less than three positive nodes, negative margins and non-aggressive histology whilst adjuvant treatment may be undertaken in those with higher risk features, including PSA persistence, three or more positive nodes, positive margins and aggressive histology. Importantly, the level of evidence for the management of pN1 patients is still low and is mainly derived from retrospective series.

Conflict of Interest: none to declare.

Funding: no funding was received for this study.

Ethics: not applicable.

\section{Legend}

Table 1. Study Features. ${ }^{* *}==$ using the exact same cohort Mayo Clinic; ${ }^{* * *}=$ using the exact same cohort University of Bern; ${ }^{* * * *}=$ using the exact same cohort San Raffaele Hospital and Mayo Clinic; $\mathrm{R}=$ retrospective series; Information Provided=reason for study inclusion, including: i) management: providing oncological 
outcomes of at least one $\mathrm{pN}+$ patients management strategy; ii) prognostic factors: providing information on prognostic factors through multivariate analysis including treatment type in case of series with multiple treatments; iii) Risk Score: providing or validating $(\mathrm{V})$ models with the aim of predicting oncological outcomes; $n \mathrm{RP}=$ number of total radical prostatectomies performed during accrual years at the Institutions involved in the study; $\mathrm{n} \mathrm{N}+$ number of $\mathrm{pN}+$ patients deriving from the number of radical prostatectomies; MFS=metastasis free survival; $\mathrm{E}=$ excluded; I=included; $\mathrm{NS}=$ not specified; $\mathrm{sRP}=$ salvage radical prostatectomy; aT=any adjuvant treatment; PSM=positive surgical margins; ${ }^{\wedge}=>0.3 \mathrm{ng} / \mathrm{mL}$ before $1994,>0.05 \mathrm{ng} / \mathrm{mL}$ before 2005 and $>0.03 \mathrm{ng} / \mathrm{mL}$ after 2005; ImageConf=Imaging confirmation; $\mathrm{BS}+\mathrm{O}=$ Bone scan plus a combination of other imaging modalities including abdominopelvic CT and/or US and/or MRI and/or chest X-ray; PET-C= Pet-choline.

Table 2. Risk of Bias and Study Quality Assessment. The Cochrane risk of bias assessment tool was used for RCTs and the Quality Appraisal tool for case series using a Modified Delphi technique for retrospective studies [15].

Table 3. Baseline features of included studies. Arms of studies providing baseline features per treatment subgroup are detailed overall and per treatment arm; ${ }^{\wedge}=$ available for 53 patients only, pre-PSA era; Abdollah et al [35] provided details of treatment arms baseline features but did not provide outcomes per treatment arm and were thus not included in Table 4. The studies of Messing et al [4,5], Moschini et al $[10,11]$, Abdollah et al $[7,33]$, Fleischman et al, although being different studies have been reported only once as they are based on exactly the same patient cohorts. *=all patients included had biochemical recurrence. Hussain et al [21] did not provide separate features of $\mathrm{pN}+$ population; '=mean (SD); ${ }^{\wedge}=$ range; $\S \S=$ all cohort, not available for the $\mathrm{pN}+$ subgroup only; In case of the same outcome being available at multiple time periods (i.e. in the same or different series in the same or different papers), the one with longest follow up/time prediction is reported [7,33], [4,5], [10,11], $[29,34]$.

Table 4. Prognostic factors analyzed in the included studies. When more categories of a single variable are reported in multivariate analysis (e.g. GleasonScore 7 vs 6 and GleasonScore 8 vs 6), the prognostic factor is considered significant if having at least one statistically significant value. Moschini et al [27] $=p T 3 b N+$ patients only. [24] Mandel et al - one or two positive nodes only. [18] ${ }^{*}$ Schiavina et al= PSA was significantly associated with worse CSS when $>40 \mathrm{mg} / \mathrm{mL} ; \quad[10]=20$ years 
estimates $^{\wedge \wedge}$; [8] and [29] and [34] and [30]=Cohorts undergoing observation only; [23]= all had negative SM; [29] and [34] same patients cohort but with different variables included in multivariate models. [31]=all patients underwent aADT; Lymphovascular invasion $(\mathrm{LVI})=$ considered present when tumor cells were found within the lumen of the blood vessels and/or nodal sinuses $;{ }^{\circ 00}=2005-20012$ vs $1987-1997$.

Table 5. Oncological Outcomes. NS=not stated; $P D=P h y s i c i a n$ Discretion; $\mathrm{OS}=$ overall Survival; $\mathrm{OD}=$ Overall Deaths; $\mathrm{CRFS}=$ clinical recurrence free survival; $\mathrm{CR}=$ Clinical Recurrence; $\mathrm{BCR}=$ Biochemical Recurrence; $\mathrm{BCRFS}=\mathrm{BCR}$ Free Survival; METS=metastasis; In case of the same outcome being available at multiple time periods (i.e. in the same or different series in the same or different papers), the one with longest follow up/time prediction is reported [7,33], [4,5], [29,34]. *=patients receiving aRT were given concomitant aADT based on physician's choice/discretion (number not specified).

\section{Supplementary Material 1. Data extracted from the included studies.}

Supplementary Material 2. Included studies by Institution. For institutions involved in multiple studies, final number of patients was calculated by considering the maximum number of patients provided by one institution in single center series; bold=number considered for the final calculation; * =study used to externally validate a nomogram = for exclusion of patients' duplicates it was assumed this study did not include patients from the institutions which developed the nomograms although some authors are present in both papers (nomogram creation and external nomogram validation). ${ }^{* *}=$ unclear if some patients were from San Raffaele hospital - the study was excluded in the calculation of the total number of patients without duplicates for this reason; also, other patients were already enrolled in Bianchi et al.

Supplementary Material 3. Cancer Specific or Overall Survival Nomograms - studies detailing nomogram creation or validation. ${ }^{*}=$ same patient cohort Cox PHR= Cox Proportional Hazard Regression; DA=discrimination accuracy; $\mathrm{n} \mathrm{N+=number}$ of positive nodes; PSM=Positive Surgical Margins; aRT= adjuvant Radiotherapy; aADT=adjuvant Androgen Deprivation Therapy; HRS=San Raffaele Hospital, Milan, Italy; Mayo Clinic= Mayo Clinic, Rochester, MI, US; MSKCC=Memorial Sloan Kettering Cancer Center, New York, NY, US; CSS= Cancer Specific Survival; OS=Overall Survival.

Supplementary Material 4. Baseline features summary of patients included in the studies reporting management of pN1 patients and their outcomes (Table 4). '=range 
of median values of the included studies/groups; "=median number of positive nodes $\mathrm{n}=$ number of studies

Supplementary file. 'A priori' study protocol. 


\begin{tabular}{|c|c|c|c|c|c|c|}
\hline Table 4. & & BCR & $\underline{\text { CR }}$ & $\underline{\text { MFS }}$ & CSS & $\underline{\text { OS }}$ \\
\hline Age & $\mathrm{Y}$ & $0.97(0.96-0.99)[26]$ & & & & $\begin{array}{l}1.04(1.02-1.06) \\
1.04(1.02-1.06) \\
1.04(1.02-1.06)\end{array}$ \\
\hline & $\mathrm{N}$ & $\begin{array}{r}1.000(0.98-1.02)[25] \\
1.00(0.99-1.01)[10] \\
0.84(0.27-1.2) \\
<65 y .0 .[23] \\
0.99(0.98-1.01)[27]\end{array}$ & $0.99(0.96-1.02)[11]$ & $\begin{array}{l}0.98(0.96-1.00)[10] \\
0.97(0.95-1.00)[25]\end{array}$ & $\begin{array}{r}1.004(0.95-1.06)[25] \\
1.00(0.95-1.05)[35] \\
0.98(0.96-1.00)[10] \\
1.00(0.97-1.02)[27] \\
1.01(0.98-1.03)[7] \\
1.00(0.98-1.04)[33] \\
0.99(0.96-1.03)[18]\end{array}$ & $0.97(0.91-$ \\
\hline PSA & $\mathrm{Y}$ & $\begin{array}{r}1.02(1.01-1.03)[24] \\
1.02(1.01-1.02)[26]\end{array}$ & & $\begin{array}{r}0.98(0.96-0.99) \text { (low } \\
\text { PSA) [8] }\end{array}$ & $\begin{array}{r}2.29(1.35-3.88)>40 \\
{[18]^{\star}}\end{array}$ & \\
\hline & $\mathrm{N}$ & $\begin{array}{r}1.2(0.91-1.56)>20 \\
{[28]} \\
1.00(1.00-1.01)[10] \\
1.01(1.00-1.02)[8] \\
1.33(0.92-1.67)<10 \\
{[23]} \\
1.00(1.00-1.00)[27] \\
1.008(0.98-1.02)[25]\end{array}$ & & $\begin{array}{r}1.000(0.99-1.01)[25] \\
\text { Categorical [28] } \\
0.99(0.97-1.02)[24] \\
1.00(1.00-1.00)[10]\end{array}$ & $\begin{array}{r}0.987(0.97-1.01)[25] \\
0.94(0.87-1.01)[24] \\
1.00(0.99-1.01)[35] \\
1.00(1.00-1.00)[10] \\
1.00(1.00-1.00)[27] \\
1.00(0.97-1.13)[33] \\
1.00(1.00-1.00)[7]\end{array}$ & $\begin{array}{l}1.00(1.00-1.00) \\
1.00(1.00-1.00) \\
1.00(1.00-1.00) \\
1.3(0.37-4.59)\end{array}$ \\
\hline GS & $\mathrm{Y}$ & $1.244(1.09-1.42)[25]$ & $2.18(1.35-3.52)>7[11]$ & $1.475(1.20-1.82)$ [25] & $\begin{array}{r}2.133(1.42-3.20)[25] \\
23.5(3.71-149)>7[24]\end{array}$ & $\begin{array}{l}2.3(1.1-5.0) \\
2.2(1.1-4.8)\end{array}$ \\
\hline
\end{tabular}




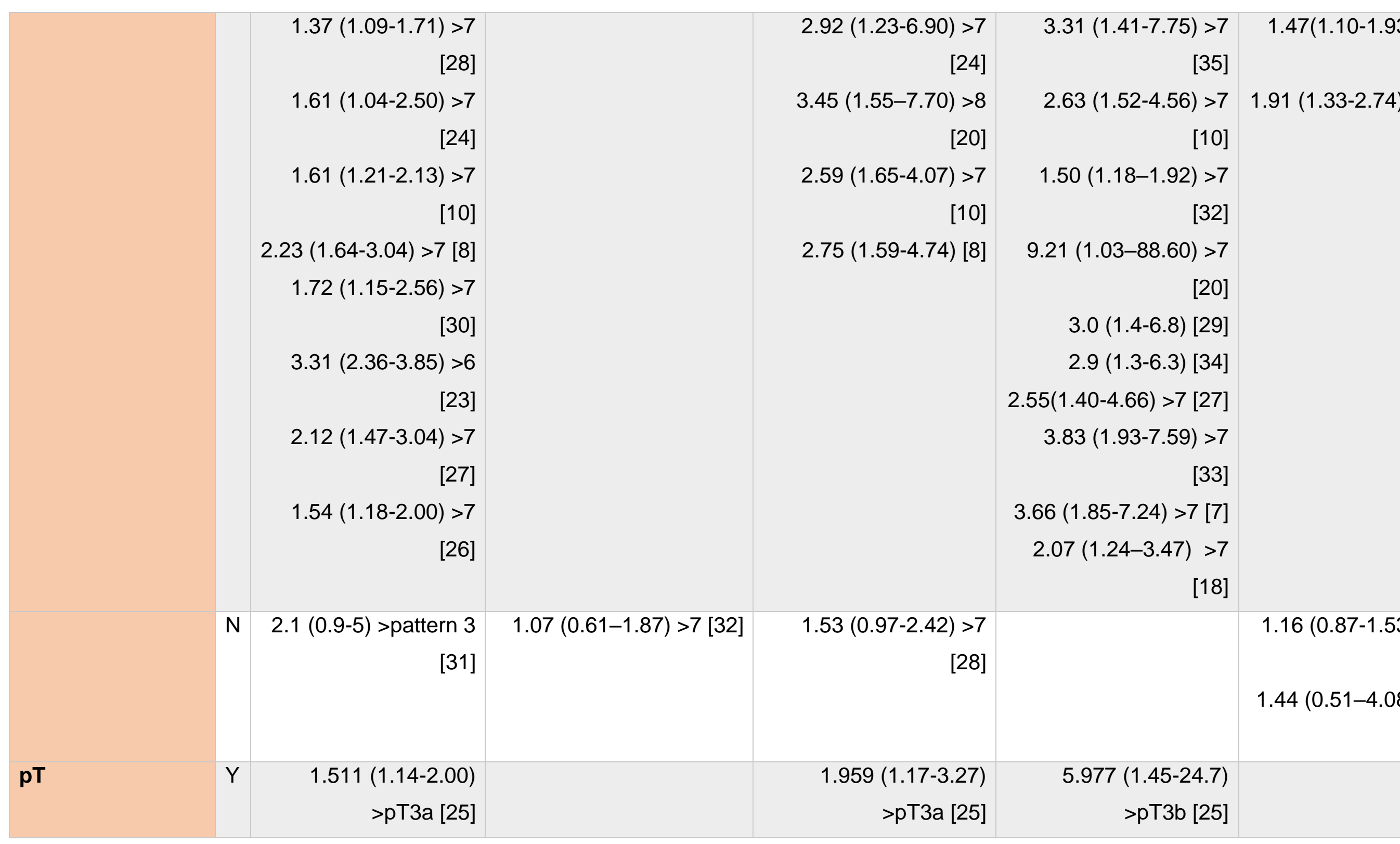




\begin{tabular}{|c|c|c|c|c|c|c|}
\hline & & $\begin{aligned} 1.71(1.16-2.51) \\
>\text { pT3a [24] } \\
1.87(1.17-2.99) \\
>\text { pT3b [10] } \\
1.87(1.11-2.04) \\
>\text { pT3a [23] } \\
2.26(1.37-3.72) \\
>\text { pT3a [26] }\end{aligned}$ & & $\begin{array}{r}2.16(1.00-4.89)>p T 3 a \\
{[24]} \\
2.17(1.18-3.99)>p \text { T3b } \\
{[10]}\end{array}$ & $\begin{array}{r}16.1(1.77-159)>\text { pT3b } \\
{[24]} \\
3.43(1.39-8.46)>\text { T3a } \\
{[20]} \\
1.91(1.12-3.25)>\text { pT3b } \\
{[33]} \\
2.37(1.15-4.88)>\text { pT3a } \\
{[7]}\end{array}$ & \\
\hline & $\mathrm{N}$ & $\begin{array}{r}1.12(0.87-1.46) \\
>\text { pT3a [28] } \\
1.33(0.86-1.84) \\
>\text { pT3a [8] } \\
1.45(0.98-2.14) \\
>\text { pT3a [30] }\end{array}$ & & $\begin{array}{r}1.48(0.81-2.73)>\text { pT3a } \\
{[28]} \\
0.91(0.53-1.56)>\text { T3a } \\
{[20]} \\
1.69(0.97-2.94)>\text { pT3a } \\
{[8]}\end{array}$ & $\begin{array}{r}0.57(0.12-2.52) \text { pT3b } \\
{[35]} \\
1.57(0.71-3.44)>\text { pT3b } \\
{[10]} \\
1.69(0.91-3.15) \\
>\text { pT3a [18] }\end{array}$ & $\begin{array}{r}1.55(0.94-\mathrm{z} \\
>\text { pT3b } \\
1.67(0.97-\mathrm{c} \\
>\text { pT3l } \\
0.7(0.25- \\
>\text { pT3 }\end{array}$ \\
\hline PSM & Y & $\begin{array}{r}1.351(1.04-1.76)[25] \\
1.39(1.08-1.78)[28] \\
1.31(1.07-1.59)[10] \\
1.41(1.15-1.73)[27] \\
1.45(1.11-1.89)[26]\end{array}$ & & $1.72(1.04-2.85)[8]$ & $\begin{array}{r}1.96(1.52-4.56)[10] \\
1.65(1.14-2.39)[27] \\
2.11(1.31-3.36)[33] \\
1.9(1.2-3.01)[7]\end{array}$ & $\begin{array}{r}1.36(1.09-) \\
1.60(1.21-2.12)\end{array}$ \\
\hline & $\mathrm{N}$ & $\begin{array}{c}1.43(0.98-2.07)[30] \\
1.24(0.85-1.80)[24] \\
1.26(0.93-1.71)[8]\end{array}$ & $\begin{array}{l}1.46(0.83-2.56)[32] \\
1.31(0.79-2.16)[11]\end{array}$ & $\begin{array}{r}1.006(0.67-1.52)[25] \\
1.01(0.61-1.67)[28] \\
1.08(0.52-2.27)[24] \\
1.32(0.98-1.79)[10]\end{array}$ & $\begin{array}{r}1.5800 .76-3.28[25] \\
1.75(0.56-5.49)[24] \\
0.92(0.4-2.13)[35] \\
1.5(0.94-2.41)[11]\end{array}$ & $\begin{array}{l}1.22(0.97- \\
1.74(0.61-\end{array}$ \\
\hline
\end{tabular}




\begin{tabular}{|c|c|c|c|c|c|c|}
\hline & & & & & $1.13(0.70-1.85)[18]$ & \\
\hline \multirow[t]{2}{*}{ N positive nodes } & $\mathrm{Y}$ & $\begin{array}{r}1.52(1.06-2.13)>2 \\
{[28]} \\
1.44(1.15-1.80)<2 \\
{[10]} \\
2.61(1.81-3.76)>2[8] \\
2.8(1.99-3.93)>2[26]\end{array}$ & $\begin{array}{l}1.03(1.01-1.05)[32] \\
1.08(1.04-1.11)[11]\end{array}$ & $\begin{array}{r}1.110(1.06-1.17)[25] \\
1.66(1.19-2.30)>2 \\
{[10]} \\
2.54(1.38-4.69)[8]\end{array}$ & $\begin{array}{r}1.137(1.05-1.23)[25] \\
1.16(1.09-1.24)[35] \\
1.75(1.78-2.61)>2[10] \\
1.1(1.05-1.16)[33] \\
1.12(1.07-1.17)[7] \\
2.16(1.32-3.52)>3 \\
{[18]}\end{array}$ & $1.08(1.03-1.13)$ \\
\hline & $\mathrm{N}$ & $\begin{array}{r}1.029(0.99-1.07)[25] \\
0.91(0.60-1.36)[24] \\
0.88(0.22-1.26)<3 \\
{[23]} \\
1.45(0.98-2.13)>1 \\
{[30]}\end{array}$ & & $\begin{array}{r}1.5(0.73-3.08)[28] \\
0.82(0.35-1.90)[24]\end{array}$ & $0.66(0.13-3.39)[24]$ & $\begin{array}{l}1.26(0.98-1.6) \\
0.6(0.16-4.08\end{array}$ \\
\hline \multirow[t]{2}{*}{$\mathrm{N}$ retrieved nodes } & $\mathrm{Y}$ & & & & $0.93(0.88-0.99)$ [35] & \\
\hline & $\mathrm{N}$ & $\begin{array}{r}1.011(1.00-1.03)[25] \\
1.01(1.00-1.02)[27] \\
1.00(0.98-1.01)[10]\end{array}$ & & $\begin{array}{r}0.997(0.97-1.02)[25] \\
1.01(0.99-1.02)[20] \\
1.00(0.99 \mathrm{e} 1.02)[10]\end{array}$ & $\begin{array}{r}0.999(0.95-1.06)[25] \\
0.97(0.94-1.01)[20] \\
1.00(0.98-1.02)[27] \\
1.03(0.96-1.05)[33] \\
1.00(0.97-1.02)[10]\end{array}$ & $\begin{array}{r}1.00(0.99-1.02) \\
1.011(0.99- \\
1.00(0.99-1.02)\end{array}$ \\
\hline
\end{tabular}




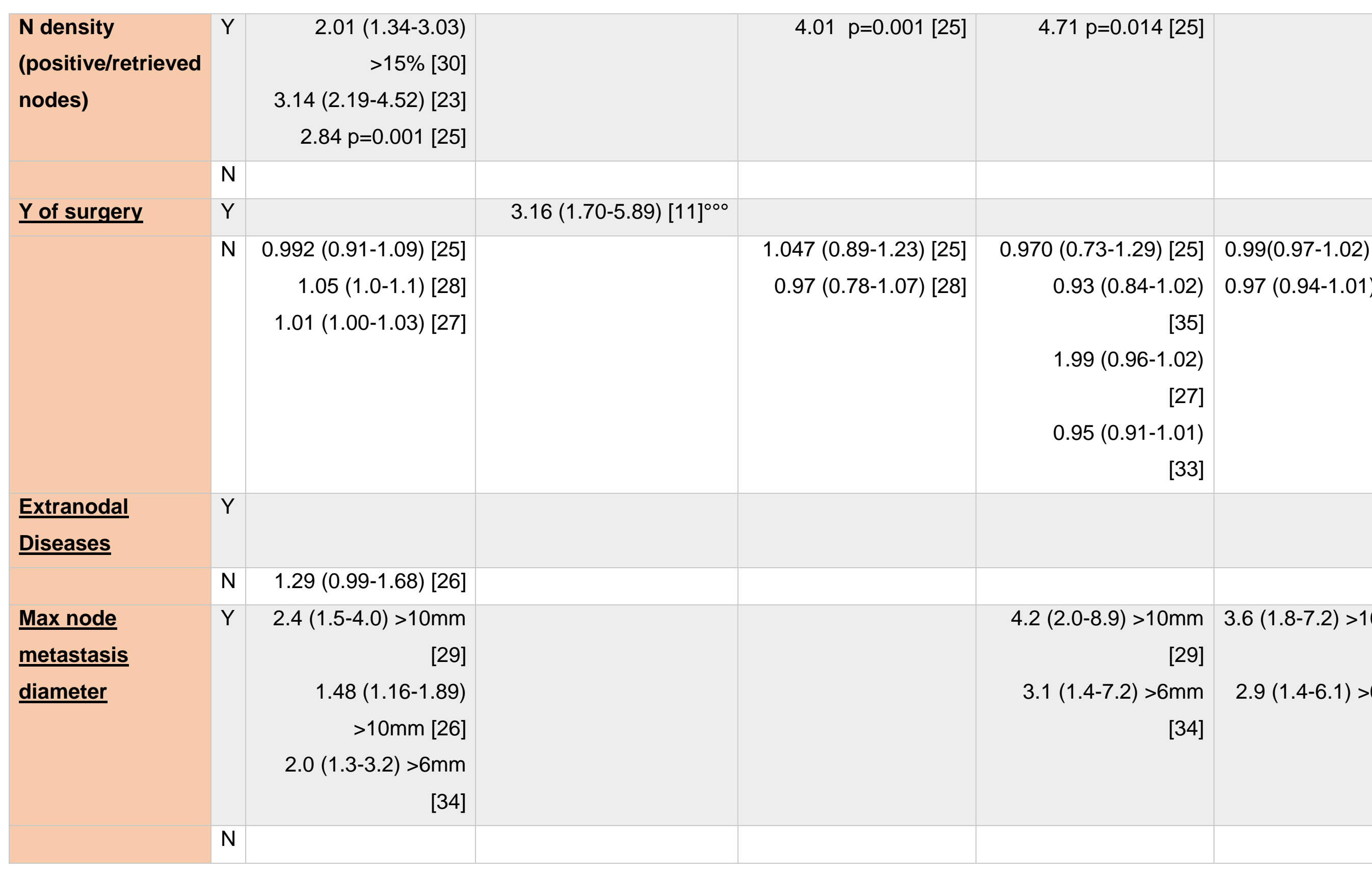




\begin{tabular}{|c|c|c|c|c|c|}
\hline \multirow[t]{2}{*}{$\begin{array}{l}\text { Node laterality } \\
\text { (mono vs } \\
\underline{\text { bilateral) }}\end{array}$} & Y & & & & \\
\hline & $\mathrm{N}$ & $3.21(1.09-3.32)[23]$ & & & \\
\hline $\begin{array}{l}\text { Lymphovascular } \\
\text { invasion in the } \\
\text { lymphnodes }\end{array}$ & $\mathrm{Y}$ & $1.9(1.1-3.5)$ [31] & & & \\
\hline & $\mathrm{N}$ & & & & \\
\hline $\begin{array}{l}\text { PSA persistence } \\
(>0.1 \mathrm{ng} / \mathrm{mL} \text { post- } \\
\text { op) }\end{array}$ & Y & & $8.34(4.73-14.72)$ [32] & & $2.16(1.63-2.86)[32]$ \\
\hline & $\mathrm{N}$ & & & & \\
\hline Time to BCR & Y & & & NS [20] & \\
\hline & $\mathrm{N}$ & & & & \\
\hline Choline-PET & Y & & & $3.45(2.14-5.56)[20]$ & \\
\hline & $\mathrm{N}$ & & & & \\
\hline $\begin{array}{l}\text { Site of } \\
\text { Recurrence }\end{array}$ & & & & & \\
\hline Skeletal & & & & & $\begin{array}{r}2.08(1.04-4.49)[20] \\
7.43(2.33-23.63)[11]\end{array}$ \\
\hline Visceral & & & & & $\begin{array}{r}4.22(1.16-11.05)[20] \\
3.13(1.36-7.22)[11]\end{array}$ \\
\hline
\end{tabular}


Multiple vs single recurrence

Time to

$1.97(1.21-3.2)$ [11]

recurrence (>5

years) 



\begin{tabular}{|c|c|c|c|c|}
\hline Institution & $\begin{array}{l}\mathrm{N} \text { of } \\
\text { studies }\end{array}$ & & & \\
\hline \multirow[t]{12}{*}{ San Raffaele Hospital } & $\mathrm{n}=11$ & & & \\
\hline & $S$ & Bravi et al & 2017 & 372 \\
\hline & $S$ & Bianchi et al & 2016 & 319 \\
\hline & $S$ & Nini et al & 2015 & 370 \\
\hline & $S$ & Abdollah et al & 2015 & 315 \\
\hline & $M$ & Moschini et al & 2016 & 1241 \\
\hline & $\mathrm{M}$ & Abdollah et al & 2014 & 1107 \\
\hline & $\mathrm{M}$ & Abdollah et al & 2014 & 1107 \\
\hline & $\mathrm{M}$ & Touijer et al & 2017 & 1388 \\
\hline & $\mathrm{M}$ & Bianchi et al & 2018 & No patients* \\
\hline & $\mathrm{M}$ & Schiavina et al & 2016 & Unclear ** \\
\hline & & Passoni et al & 2013 & 484 \\
\hline \multirow[t]{7}{*}{ Mayo Clinic } & $n=6$ & & & \\
\hline & S & Moschini et al & 2015 & $\underline{1011}$ \\
\hline & $S$ & Moschini et al & 2015 & 1011 \\
\hline & $\mathrm{M}$ & Moschini et al & 2016 & 1241 \\
\hline & $\mathrm{M}$ & Abdollah et al & 2014 & 1107 \\
\hline & $\mathrm{M}$ & Abdollah et al & 2014 & 1107 \\
\hline & $\mathrm{M}$ & Touijer et al & 2017 & 1388 \\
\hline \multirow[t]{5}{*}{ Martini Klinik } & $\mathrm{n}=4$ & & & \\
\hline & $S$ & Mandel et al & 2017 & 706 \\
\hline & $S$ & Tilki et al & 2017 & 773 \\
\hline & $S$ & Mandel et al & 2017 & 268 \\
\hline & $\mathrm{M}$ & Passoni et al & 2013 & 484 \\
\hline \multicolumn{5}{|l|}{ MSKCC } \\
\hline & $S$ & Touijer et al & 2013 & 369 \\
\hline & $\mathrm{M}$ & Touijer et al & 2017 & 1388 \\
\hline \multicolumn{5}{|l|}{ University of Bern } \\
\hline & $S$ & $\begin{array}{l}\text { Fleischman et } \\
\text { al }\end{array}$ & 2009 & 102 \\
\hline & $\mathrm{S}$ & & 2008 & 102 \\
\hline
\end{tabular}




\begin{tabular}{|l|l|l|l|l|}
\hline $\begin{array}{l}\text { Other series not } \\
\text { including duplicates }\end{array}$ & & & & \\
\hline & S & Dorin et al & 2012 & $\mathbf{1 5 0}$ \\
\hline & S & Hofer et al & 2006 & $\mathbf{2 1 1}$ \\
\hline & S & Palapattu et al & 2004 & $\mathbf{1 1 9}$ \\
\hline & M & Cai et al & 2009 & $\mathbf{1 2 4}$ \\
\hline & M & Bianchi et al & 2018 & $\mathbf{5 7 6}$ \\
\hline & M & Messing & 2006 & $\mathbf{9 8}$ \\
\hline & M & Hussain & 2018 & $\mathbf{1 6 2}$ \\
\hline
\end{tabular}

\section{Supplementary Material 2.}

\section{REFERENCES}

[1] Abdollah F, Suardi N, Gallina A, Bianchi M, Tutolo M, Passoni N, et al. Extended pelvic lymph node dissection in prostate cancer: A 20-year audit in a single center. Ann Oncol 2013;24:1459-66. doi:10.1093/annonc/mdt120.

[2] Bernstein AN, Shoag JE, Golan R, Halpern JA, Schaeffer EM, Hsu WC, et al. Contemporary Incidence and Outcomes of Prostate Cancer Lymph Node Metastases. J Urol 2018;199:1510-7. doi:10.1016/j.juro.2017.12.048.

[3] Parker C, Clarke NW, Cook A et al. Timing of radiotherapy after radical prostatectomy. ESMO Congr 2019 Abstr LBA49_PR Present Sept 27, 2019 n.d.

[4] Messing EM, Manola J, Yao J, Kiernan M, Crawford D, Wilding G, et al. Immediate versus deferred androgen deprivation treatment in patients with node-positive prostate cancer after radical prostatectomy and pelvic lymphadenectomy. Lancet Oncol 2006;7:472-9. doi:10.1016/S1470-2045(06)70700-8.

[5] Messing, Eduard M.; Manola Judith M.S.; Trump DMD. After Radical Prostatectomy and Pelvic Lymphadenectomy in Men With Node-Positive Prostate Cancer. N Engl J Med 1999;341:1781-8.

[6] Preisser F, Marchioni M, Nazzani S, Bandini M, Tian Z, Pompe RS, et al. The Impact of Lymph Node Metastases Burden at Radical Prostatectomy. Eur Urol Focus 
2019;5:399-406. doi:10.1016/j.euf.2017.12.009.

[7] Abdollah F, Karnes RJ, Suardi N, Cozzarini C, Gandaglia G, Fossati N, et al. J OURNAL OF C LINICAL O NCOLOGY Impact of Adjuvant Radiotherapy on Survival of Patients With Node-Positive Prostate Cancer 2014:1-10. doi:10.1200/JCO.2013.54.7893.

[8] Touijer KA, Mazzola CR, Sjoberg DD, Scardino PT, Eastham JA. Long-term outcomes of patients with lymph node metastasis treated with radical prostatectomy without adjuvant androgen-deprivation therapy. Eur Urol 2014;65:20-5. doi:10.1016/j.eururo.2013.03.053.

[9] Touijer KA, Karnes RJ, Passoni N, Sjoberg DD, Assel M, Fossati N, et al. Survival Outcomes of Men with Lymph Node-positive Prostate Cancer After Radical Prostatectomy: A Comparative Analysis of Different Postoperative Management Strategies. Eur Urol 2018;73:890-6. doi:10.1016/j.eururo.2017.09.027.

[10] Moschini M, Sharma V, Zattoni F, Boorjian SA, Frank I, Gettman MT, et al. Risk Stratification of $\mathrm{pN}+$ Prostate Cancer after Radical Prostatectomy from a Large Single Institutional Series with Long-Term Followup. J Urol 2016;195:1773-8. doi:10.1016/j.juro.2015.12.074.

[11] Moschini M, Sharma V, Zattoni F, Quevedo JF, Davis BJ, Kwon E, et al. Natural History of Clinical Recurrence Patterns of Lymph Node-Positive Prostate Cancer after Radical Prostatectomy. Eur Urol 2016;69:135-42. doi:10.1016/j.eururo.2015.03.036.

[12] Zareba P, Eastham J, Scardino PT, Touijer K. Contemporary Patterns of Care and Outcomes of Men Found to Have Lymph Node Metastases at the Time of Radical Prostatectomy. J Urol 2017;198:1077-84. doi:10.1016/j.juro.2017.06.062.

[13] Passoni M, D M, Abdollah F, D M, Suardi N, D M, et al. Head-to-head comparison of lymph node density and number of positive lymph nodes in stratifying the outcome of patients with lymph node-positive prostate cancer submitted to radical prostatectomy and extended lymph node dissection 2014;32. doi:10.1016/j.urolonc.2012.10.009.

[14] Knoll T, Omar MI, Maclennan S, Hernández V, Canfield S, Yuan Y, et al. Key Steps in Conducting Systematic Reviews for Underpinning Clinical Practice Guidelines: Methodology of the European Association of Urology. Eur Urol 2018;73:290-300. doi:10.1016/j.eururo.2017.08.016.

[15] Moga C, Guo B, Schopflocher D HC. Development of a Quality Appraisal Tool for Case Series. Stud Using a Modif Delphi Tech 2012. 
[16] Ploussard G, Gandaglia G, Borgmann H, de Visschere P, Heidegger I, Kretschmer A, et al. Salvage Lymph Node Dissection for Nodal Recurrent Prostate Cancer: A Systematic Review. Eur Urol 2019;76:493-504. doi:10.1016/j.eururo.2018.10.041.

[17] Mitropoulos D, Artibani W, Graefen M, Remzi M, Rouprêt M, Truss M. Reporting and Grading of Complications After Urologic Surgical Procedures: An ad hoc EAU Guidelines Panel Assessment and Recommendations. Eur Urol 2012;61:341-9. doi:10.1016/j.eururo.2011.10.033.

[18] Schiavina R, Bianchi L, Borghesi M, Briganti A, Brunocilla E, Carini M, et al. Original Article : Clinical Investigation Predicting survival in node-positive prostate cancer after open , laparoscopic or robotic radical prostatectomy : A competing risk analysis of a multi-institutional database 2016. doi:10.1111/iju.13203.

[19] Bianchi L, Schiavina R, Borghesi M, Bianchi FM, Briganti A, Carini M, et al. Evaluating the predictive accuracy and the clinical benefit of a nomogram aimed to predict survival in node-positive prostate cancer patients: External validation on a multi-institutional database. Int J Urol 2018;25:574-81. doi:10.1111/iju.13565.

[20] Nini A, Gandaglia G, Fossati N, Suardi N, Cucchiara V, Dell'Oglio P, et al. Patterns of clinical recurrence of node-positive prostate cancer and impact on long-term survival. Eur Urol 2015;68:777-84. doi:10.1016/j.eururo.2015.04.035.

[21] Hussain M, Tangen CM, Thompson IM, Swanson GP, Wood DP, Sakr W, et al. Phase III intergroup trial of adjuvant androgen deprivation with or without mitoxantrone plus prednisone in patients with high-risk prostate cancer after radical prostatectomy: SWOG S9921. J Clin Oncol 2018;36:1498-504. doi:10.1200/JCO.2017.76.4126.

[22] Bravi CA, Tin A, Vertosick E, Mazzone E, Bandini M, Dell'Oglio P, et al. Androgen deprivation therapy in men with node-positive prostate cancer treated with postoperative radiotherapy. Urol Oncol Semin Orig Investig 2019;000. doi:10.1016/j.urolonc.2019.09.018.

[23] Cai T, Nesi G, Ph D, Tinacci G, Giubilei G, Gavazzi A, et al. Clinical Importance of Lymph Node Density in Predicting Outcome of Prostate Cancer Patients. J Surg Res 2011;167:267-72. doi:10.1016/j.jss.2009.05.004.

[24] Mandel P, Rosenbaum C, Pompe RS, Steuber T, Salomon G, Chun FK, et al. Longterm oncological outcomes in patients with limited nodal disease undergoing radical prostatectomy and pelvic lymph node dissection without adjuvant treatment. World J Urol 2017;35:1833-9. doi:10.1007/s00345-017-2079-4. 
[25] Mandel P, Kriegmair MC, Bogdan K, Boehm K, Budäus L, Graefen M, et al. Association between Lymph Node Counts and Oncological Outcomes in Lymph Node Positive Prostate Cancer. Eur Urol Focus 2017;3:248-55. doi:10.1016/j.euf.2016.02.018.

[26] Passoni NM, Fajkovic H, Xylinas E, Kluth L, Seitz C, Robinson BD, et al. Prognosis of patients with pelvic lymph node (LN) metastasis after radical prostatectomy: Value of extranodal extension and size of the largest LN metastasis. BJU Int 2014;114:503-10. doi:10.1111/bju.12342.

[27] Moschini M, Sharma V, Gandaglia G, Dell'Oglio P, Fossati N, Zaffuto E, et al. Longterm utility of adjuvant hormonal and radiation therapy for patients with seminal vesicle invasion at radical prostatectomy. BJU Int 2017;120:69-75. doi:10.1111/bju.13683.

[28] Tilki D, Preisser F, Tennstedt P, Tober P, Mandel P, Schlomm T, et al. Adjuvant radiation therapy is associated with better oncological outcome compared with salvage radiation therapy in patients with $\mathrm{pN} 1$ prostate cancer treated with radical prostatectomy. BJU Int 2017;119:717-23. doi:10.1111/bju.13679.

[29] Fleischmann A, Schobinger S, Schumacher M, Thalmann GN, Studer UE. Survival in SurgicallyT reated, Nodal Positive Prostate Cancer Patients Is Predicted by Histopathological Characteristics of the PrimaryT umor and Its Lymph Node Metastases 2009;362. doi:10.1002/pros.20889.

[30] Palapattu GS, Allaf ME, Trock BJ, Epstein JI, Walsh PC. PROSTATE SPECIFIC ANTIGEN PROGRESSION IN MEN WITH LYMPH NODE METASTASES FOLLOWING RADICAL PROSTATECTOMY : RESULTS OF LONG-TERM FOLLOWUP 2004;172:1860-4. doi:10.1097/01.ju.0000139886.25848.4a.

[31] Hofer MD, Kuefer R, Huang WEI, Li H, Bismar TA, Perner S, et al. PROGNOSTIC FACTORS IN LYMPH NODE-POSITIVE 2005. doi:10.1016/j.urology.2005.10.055.

[32] Bianchi L, Nini A, Bianchi M, Gandaglia G, Fossati N, Suardi N, et al. The Role of Prostate-specific Antigen Persistence after Radical Prostatectomy for the Prediction of Clinical Progression and Cancer-specific Mortality in Node-positive Prostate Cancer Patients. Eur Urol 2016;69:1142-8. doi:10.1016/j.eururo.2015.12.010.

[33] Abdollah F, Jeffrey Karnes R, Suardi N, Cozzarini C, Gandaglia G, Fossati N, et al. Predicting survival of patients with node-positive prostate cancer following multimodal treatment. Eur Urol 2014;65:554-62. doi:10.1016/j.eururo.2013.09.025.

[34] Fleischmann A, Schobinger S, Markwalder R, Schumacher M, Burkhard F, 
Thalmann GN. Prognostic factors in lymph node metastases of prostatic cancer patients : the size of the metastases but not extranodal extension independently predicts survival 2008:468-75. doi:10.1111/j.1365-2559.2008.03129.x.

[35] Abdollah F, Gandaglia G, Suardi N, Capitanio U, Salonia A, Nini A, et al. More extensive pelvic lymph node dissection improves survival in patients with nodepositive prostate cancer. Eur Urol 2015;67:212-9. doi:10.1016/j.eururo.2014.05.011.

[36] Dorin RP, Lieskovsky G, Fairey AS, Cai J, Daneshmand S. Outcomes after radical prostatectomy for patients with clinical stages T1-T2 prostate cancer with pathologically positive lymph nodes in the prostate-specific antigen era . URO 2013;31:1441-7. doi:10.1016/j.urolonc.2012.03.006.

[37] Neal DE, Metcalfe C, Donovan JL, Lane JA, Davis M, Young GJ, et al. Ten-year Mortality, Disease Progression, and Treatment-related Side Effects in Men with Localised Prostate Cancer from the ProtecT Randomised Controlled Trial According to Treatment Received. Eur Urol 2019:1-11. doi:10.1016/j.eururo.2019.10.030.

[38] Yang DD, Mahal BA, Muralidhar V, Martin NE, Orio PF, Mouw KW, et al. Androgen Deprivation Therapy and Overall Survival for Gleason 8 Versus Gleason 9-10 Prostate Cancer. Eur Urol 2019;75:35-41. doi:10.1016/j.eururo.2018.08.033.

[39] Preisser F, Coxilha G, Heinze A, Oh S, Chun FK-H, Sauter G, et al. Impact of positive surgical margin length and Gleason grade at the margin on biochemical recurrence in patients with organ-confined prostate cancer. Prostate 2019. doi:10.1002/pros.23908.

[40] Moris L, Cumberbatch MG, Van den Broeck T, Gandaglia G, Fossati N, Kelly B, et al. Benefits and Risks of Primary Treatments for High-risk Localized and Locally Advanced Prostate Cancer: An International Multidisciplinary Systematic Review. Eur Urol 2020;7. doi:10.1016/j.eururo.2020.01.033.

[41] Tilki D, Pompe RS, Bandini M, Marchioni M, Kretschmer A, Tian Z, et al. Local treatment for metastatic prostate cancer: A systematic review. Int J Urol 2018;25:390-403. doi:10.1111/iju.13535.

[42] Vale CL, Brihoum M, Chabaud S et al. Adjuvant or salvage radiotherapy for the treatment of localised prostate cancer? ESMO Congr 2019 Abstr LBA48_PR Present Sept 27, 2019 n.d.

[43] Hofman MS, Lawrentschuk N, Francis RJ, Tang C, Vela I, Thomas P, et al. Prostatespecific membrane antigen PET-CT in patients with high-risk prostate cancer before curative-intent surgery or radiotherapy (proPSMA): a prospective, randomised, multi- 
centre study. Lancet 2020;395:1208-16. doi:10.1016/s0140-6736(20)30314-7.

[44] Dalela D, Santiago-Jiménez M, Yousefi K, Karnes RJ, Ross AE, Den RB, et al. Genomic classifier augments the role of pathological features in identifying optimal candidates for adjuvant radiation therapy in patients with prostate cancer:

Development and internal validation of a multivariable prognostic model. J Clin Oncol 2017;35:1982-90. doi:10.1200/JCO.2016.69.9918.

[45] Gore JL, du Plessis M, Santiago-Jiménez M, Yousefi K, Thompson DJS, Karsh L, et al. Decipher test impacts decision making among patients considering adjuvant and salvage treatment after radical prostatectomy: Interim results from the Multicenter Prospective PRO-IMPACT study. Cancer 2017;123:2850-9.

doi:10.1002/cncr.30665. 